\title{
Wavelet-based spatial comparison technique for analysing and evaluating two-dimensional geophysical model fields
}

\author{
S. Saux Picart, M. Butenschön, and J. D. Shutler \\ Plymouth Marine Laboratory, Prospect Place, The Hoe, PL1 3DH Plymouth, UK \\ Correspondence to: S. Saux Picart (stux@pml.ac.uk) \\ Received: 26 October 2011 - Published in Geosci. Model Dev. Discuss.: 24 November 2011 \\ Revised: 2 February 2012 - Accepted: 8 February 2012 - Published: 13 February 2012
}

\begin{abstract}
Complex numerical models of the Earth's environment, based around 3-D or 4-D time and space domains are routinely used for applications including climate predictions, weather forecasts, fishery management and environmental impact assessments. Quantitatively assessing the ability of these models to accurately reproduce geographical patterns at a range of spatial and temporal scales has always been a difficult problem to address. However, this is crucial if we are to rely on these models for decision making. Satellite data are potentially the only observational dataset able to cover the large spatial domains analysed by many types of geophysical models. Consequently optical wavelength satellite data is beginning to be used to evaluate model hindcast fields of terrestrial and marine environments. However, these satellite data invariably contain regions of occluded or missing data due to clouds, further complicating or impacting on any comparisons with the model. This work builds on a published methodology, that evaluates precipitation forecast using radar observations based on predefined absolute thresholds. It allows model skill to be evaluated at a range of spatial scales and rain intensities. Here we extend the original method to allow its generic application to a range of continuous and discontinuous geophysical data fields, and therefore allowing its use with optical satellite data. This is achieved through two major improvements to the original method: (i) all thresholds are determined based on the statistical distribution of the input data, so no a priori knowledge about the model fields being analysed is required and (ii) occluded data can be analysed without impacting on the metric results. The method can be used to assess a model's ability to simulate geographical patterns over a range of spatial scales. We illustrate how the method provides a compact and concise way of visualising the degree of agreement between spatial features in two datasets. The application of the new method, its handling of bias and occlusion and the advantages of the novel method are demonstrated through the analysis of model fields from a marine ecosystem model.
\end{abstract}

\section{Introduction}

Numerical models of the environment are now widely used in a large number of applications. Recent topical examples include modelling the movement of ash clouds (e.g. Jones et al., 2007) from the 2010 eruption of the volcano Eyjafjallajökull in Iceland which impacted on world wide air travel and modelling the path of the Deep-water Horizon oil spill in the Gulf of Mexico (e.g. Lehr et al., 2000), towards guiding the environmental clean up operations. In the context of marine ecosystems, models are used for a number of applications including climate predictions, fishery and coastal management and environmental impact assessment. As these models increase in complexity and our reliance on them increases, so does the need to assess the accuracy of their predictions. The development of methodological approaches to assess the skill of geophysical model predictions has been a prominent subject for a number of scientific publications, leading to a range of different techniques usually involving the comparison of two independent datasets. Many works, particularly in the context of precipitation forecasts and more recently for hydrodynamic-ecosystem models, have shown the importance of using a suite of metrics (Bougeault, 2003; Ebert et al., 2003; Allen et al., 2007; Doney et al., 2009; Stow et al., 2009) along with the need to study different temporal and spatial scales (Tiedje et al., 2010; Shutler et al., 2011). Many of these approaches have studied categorical and continuous verification approaches which include metrics related to bias, variability and correlations between the two datasets being studied. The use of multiple metrics aids the identification of differences between the two datasets, while providing insights into the causes of the observed differences. Many of the published metric techniques are based on time series analysis assessing the data using point to point comparisons and aggregation using arbitrary or user defined spatial and temporal scales. However, most applications of these models require the representation of specific geophysical features, 
with specific space and time scales, which may vary considerably between applications and will depend upon the data that is being analysed. To fully assess these models the identification of the model skill over a range of spatial and temporal scales is crucial. Additionally, allowing the distribution of the data being analysed to guide the setting of any aggregation levels would allow approaches to be more generic. Relatively recent work in the field of precipitation forecast analysis has seen the development of techniques for studying two-dimensional binary difference maps using Haar wavelets (Casati et al., 2004; Casati, 2010). This work is itself based on an earlier study from Briggs and Levine (1997) who used wavelet decomposition in field forecast verification. The binary maps, defined for specific thresholds of the geophysical dataset, are the result of differencing the two input datasets, while the use of the Haar wavelet allows the identification of the orthogonal spatial structures responsible for any differences. Haar wavelets (Haar, 1910) are discontinuous and are therefore suitable for handling spatially discontinuous data fields. The approach of Casati et al. (2004) was recently applied to analysing the performance of a hydrodynamic ecosystem model (Shutler et al., 2011). In both situations, the thresholds of the different parameters used to generate the binary difference maps were manually set, based on user experience, and therefore the evaluation results are likely to vary with respect to the thresholds chosen.

Satellite or Earth observation data provide an excellent dataset to evaluate model fields. Indeed, Earth observation is one of the few sources of data that can provide the required spatially-continuous datasets needed to evaluate the outputs of large spatial coverage geophysical models. Visible and infrared remote sensing data can be used to evaluate global marine hydrodynamic ecosystems models (Shutler et al., 2011) through two major variables: chlorophyll- $a$ surface concentration and sea surface temperature. However, visible (spectral wavelengths between $400-600 \mathrm{~nm}$ ) and infrared (spectral wavelengths between 700-1000 nm) fields of the oceans measured from a satellite will invariably contain occluded or missing data due to clouds (e.g. the optical sensor is unable to see through cloud). This can present a problem when using these data to evaluate model fields as (in contrast) the model fields will be spatially complete. Removing the equivalent data from the model data before comparison with the Earth observation data (e.g. as done by Shutler et al., 2011) is a simple way of addressing that issue. However, dependent upon the dataset, this can have a significant impact upon the statistical distribution of the dataset being analysed, and thus can potentially impact on any evaluation results.

In this paper, the original method of Casati et al. (2004) has been extended to handle regions of missing or occluded data, while maintaining the orthogonality of the wavelet approach. Furthermore, to make the methodology more objective and to enable the generic application of the approach to alternative applications (e.g. other geophysical models), the thresholds are determined based on the statistical distribution of each input dataset. This produces a comparison of the spatial structures inherent to each dataset (as shall be illustrated below) comparing extremes of one set to extremes of the other and average conditions to average conditions. To illustrate its application this new approach has been applied to assess the performance of important state variables of a dynamic marine ecosystem model, comparing the output to data derived from satellite Earth observation. The technique is equally applicable to alternative scenarios including evaluating the performance of precipitation and climate forecast models. The paper is structured as follows. Section 2 gives a description of the methodology developed as well as an overview of the original methodology of Casati et al. (2004), highlighting the novel enhancements. Section 3 illustrates its application, followed by a discussion about the benefits offered. Section 4 gives a summary of the methodology along with possible applications.

\section{Methodology}

The methodology we propose here evaluates the match of two-dimensional representations of two datasets at distinct spatial scales through wavelet decomposition. This section gives a brief overview of the original methodology of Casati et al. (2004) and a detailed description of the novel extensions.

\subsection{Overview of original method}

The original methodology was developed by Casati et al. (2004) for verifying spatial precipitation forecasts. It consists of a suite of simple operations carried out on a set of user-defined thresholds of the variable of interest. A metric comparing spatial maps based on these thresholds (or cutoffs) then summarises the ability of a model to simulate the geophysical structures under investigation. The different steps of this process for a particular threshold are described briefly:

- Computing the binary fields for the two datasets, respectively: for a given threshold $t$ and a data field $\mathbf{D}$, the binary image $\mathbf{I}$ is defined by: $\mathbf{I}=1$ where $\mathbf{D} \geq t$ and $\mathbf{I}=0$ where $\mathbf{D}<t$.

- Computing the binary difference map: subtraction of the corresponding binary fields.

- Performing a 2-D-Haar wavelet decomposition on the binary difference map.

- Computing the mean square error and skill score for each level of decomposition. 


\subsection{Enhanced method}

The method outlined above allowed the authors to evaluate the forecast skill as a function of precipitation rate and spatial scale. Shutler et al. (2011) applied the method for evaluating the performance of a hydrodynamic-ecosystem model. However, occluded data was handled very simply resulting in a loss of orthogonality hence skills at scales subject to occlusion were affected by smaller scale errors. Additionally, the thresholds used to generate the binary maps were set at arbitrary absolute levels.

A modified version of this wavelet analysis is hereafter presented in generic terms.

\subsubsection{Binary difference maps}

The whole methodology is based on the concept of binary difference maps. The degradation of the continuous field to a binary map is a crucial step as it defines the patterns in the datasets that are going to be compared. Instead of using absolute thresholds to define the binary difference image (as was used in the original methodology by Casati et al., 2004), we apply the methodology over ranges inherent to the data sets as suggested by Yates et al. (2006). These ranges are defined by the quantiles of the data distribution, evaluated for each of the two datasets independently. For example, if we consider the variable $V$, we may define quantiles $V^{0 \%}=$ $V_{\min } ; V^{20 \%} ; V^{40 \%} ; V^{60 \%} ; V^{80 \%}$ and $V^{100 \%}=V_{\max }$. These quantiles can then be used to define five intervals in each of the datasets: $\left[V^{0 \%}, V^{20 \%}\right),\left[V^{20 \%}, V^{40 \%}\right) ;\left[V^{40 \%}, V^{60 \%}\right)$; $\left[V^{60 \%}, V^{80 \%}\right) ;\left[V^{80 \%}, V^{100 \%}\right]$. The methodology allows for any number of quantiles. However, here for simplicity we have chosen to use the five ranges defined above.

Considering two 2-D spatial fields $\mathbf{X}$ and $\mathbf{Y}$, and following the notation of Shutler et al. (2011) we define the binary masks for the two data fields $\left(\mathbf{I}_{Y}\right)$ and $\left(\mathbf{I}_{X}\right)$ by:

$$
\begin{gathered}
\mathbf{I}_{X}= \begin{cases}1, & X^{q_{1}} \leq \mathbf{X}<X^{q_{2}} \\
0, & \text { else }\end{cases} \\
\mathbf{I}_{Y}=\left\{\begin{array}{ll}
1, & Y^{q_{1}} \leq \mathbf{Y}<Y^{q_{2}} \\
0, & \text { else }
\end{array},\right.
\end{gathered}
$$

where $X^{q_{1}}, X^{q_{2}}\left(Y^{q_{1}}, Y^{q_{2}}\right.$ respectively) are two consecutive quantiles for each dataset, defining what we will refer to, in the following, as $q$, quantile range ([ $\left[X^{q_{1}}, X^{q_{2}}\right)$ and $\left[Y^{q_{1}}, Y^{q_{2}}\right)$, respectively). We note here that if we chose equally-spaced quantiles the number of data points attributed to each range would be identical for both data fields. This is an important improvement with respect to the original methodology because it allows the study of inherent patterns in the two images, removing the need for absolute thresholds values.
From these two binary masks we then compute the binary difference map $\mathbf{Z}$, defined by $\mathbf{Z}=\mathbf{I}_{Y}-\mathbf{I}_{X}$, and noted $\mathbf{Z}_{q}$ when referring to the quantile range $q$.

Figure 1 illustrates the process of creating a binary difference map for ocean chlorophyll- $a$ data obtained from model and satellite imagery. In the left column of Fig. 1 are the satellite estimates (top), and the model estimates (bottom). From these two fields, quantile maps are derived (second column on the same figure), that show the patterns associated with the quantile definition. By subtracting these two maps we obtain the binary difference map (right-hand side map on Fig. 1) which is fed into the wavelet decomposition described in the next section (Sect. 2.2.2).

\subsubsection{Wavelet decomposition}

The binary difference map as defined above is decomposed using an improved wavelet decomposition technique with respect the original one presented by Casati et al. (2004). We introduce into the wavelet decomposition a weight image $\zeta_{0}$ that reduces the impact of heavily occluded areas on the difference metrics while preserving the orthogonality between the scale components:

$\zeta_{0}= \begin{cases}1 & \text { for valid data } \\ 0 & \text { for missing data }\end{cases}$

As described by Casati et al. (2004), a two-dimensional discrete Haar wavelet decomposition can be performed by spatially averaging over a $2^{l} \times 2^{l}$ pixel region, where $l$ is the level of decomposition. We define the $l$-th father $\left(\mathbf{W}_{\text {father }}^{l}\right)$ and mother $\left(\mathbf{W}_{\text {mother }}^{l}\right)$ wavelet component by:

$$
\begin{aligned}
\mathbf{W}_{\text {father }}^{l}\left(\mathbf{Z}_{q}\right) & =\frac{\left\langle\mathbf{Z}_{q} \zeta_{0}\right\rangle_{2^{l} \times 2^{l}}}{\left\langle\zeta_{0}\right\rangle_{2^{l}} \times 2^{l}} \\
\mathbf{W}_{\text {mother }}^{l}\left(\mathbf{Z}_{q}\right) & =\mathbf{W}_{\text {father }}^{l-1}\left(\mathbf{Z}_{q}\right)-\mathbf{W}_{\text {father }}^{l}\left(\mathbf{Z}_{q}\right)
\end{aligned}
$$

where the notation $\langle\cdot\rangle_{2^{l} \times 2^{l}}$ refers to a $2^{l} \times 2^{l}$ spatial averaging. The $l$-th father wavelet component is obtained by spatial averaging over $2^{l} \times 2^{l}$ pixels and is therefore a smoothed representation of the original binary difference map. The $l$-th mother wavelet quantifies the differences between the original binary difference map and the average generated by the father wavelet.

This decomposition is done retaining the original resolution of the image, thus allowing to use the same weight image for each aggregation level. This formulation maintains the orthogonality and conserves the original signal contained in the split components, i.e.

$\mathbf{Z}_{q}=\mathbf{W}_{\text {father }}^{L}\left(\mathbf{Z}_{q}\right)+\sum_{l=1}^{L} \mathbf{W}_{\text {mother }}^{l}\left(\mathbf{Z}_{q}\right)$

where $L$ is the upper level of decomposition. 

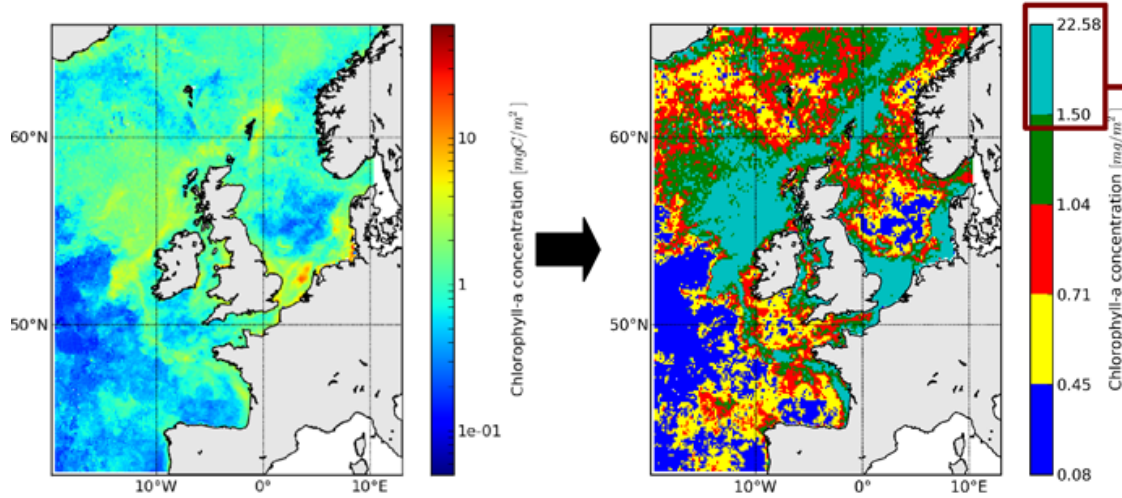
for the $5^{\text {th }}$ quantile range
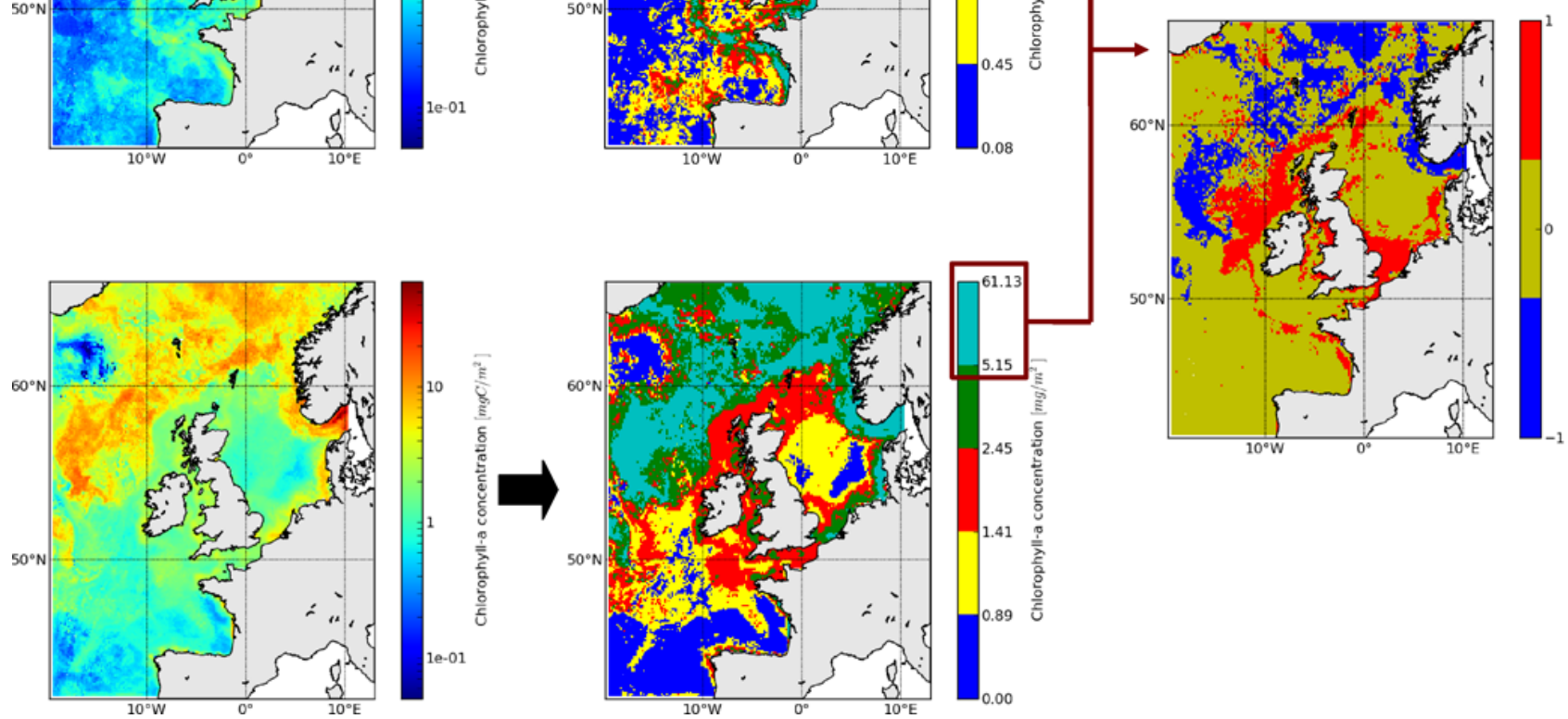

Fig. 1. Binary difference map creation. On the left: re-gridded satellite (top) and model (bottom) monthly fields of surface concentration of chlorophyll- $a$ for May 2004. In the centre: quantile maps of the same fields (top, satellite; bottom model). On the right: binary difference map for the uppermost quantile range.

\subsubsection{Mean squared differences and skill score}

For each level of decomposition $(l)$ and each quantile $(q)$, the mean squared difference of the mother wavelet $\left(\mathrm{MSE}_{l, q}\right)$ is computed by:

$\operatorname{MSE}_{l, q}=\frac{\sum\left[\left(\mathbf{W}_{\text {mother }}^{l}\left(\mathbf{Z}_{q}\right) \zeta_{0}\right)^{2}\right]}{\sum \zeta_{0}}$

where $\sum$ means summation over the whole domain. The inclusion of $\zeta_{0}$ allows any missing or occluded data to be accounted for.

The overall mean squared difference is maintained through the decomposition and the following equation remains true:

$\operatorname{MSE}_{q}=\sum_{l=1}^{L} \operatorname{MSE}_{l, q}$

where $\mathrm{MSE}_{q}$ refers to the overall mean squared difference of the binary difference map.
We then compute the skill score (SS) as defined in Casati et al. (2004) which is more intuitive to interpret than the MSE: 1 means a perfect match, 0 corresponds to the comparison of random data, below 0 represents a match worse than due to random chance alone. The formulation of the skill score is as follow:

$\mathrm{SS}_{l, q}=1-\frac{\mathrm{MSE}_{l, q} L}{2 \varepsilon_{q}\left(1-\varepsilon_{q}\right)}$

where $\varepsilon_{q}$ is the fraction of data contained in the quantile $q$. The skill score is in fact defined as the mean square error relative to the means square error of a random no skill simulation (see Casati et al., 2004).

\section{Results and discussion}

In this section we demonstrate how the wavelet analysis can be used to interpret the differences between model and satellite fields. The methodology is applied to study the case of chlorophyll- $a$ and SST in the North East Atlantic European shelf sea. 


\subsection{Satellite data and hydrodynamic-ecosystem model}

To accommodate the reader we give brief introductions to the data sets used in the examples. We shall not go into the details of the geophysical application and the implications of the skill assessment, but rather provide a quick overview to enable the reader to fully understand the methodology and its benefits. The data shown serve simply as examples to provide a show case for the methodology.

The model used in this work is an implementation of the POLCOMS-ERSEM model (Allen et al., 2001, 2007) for the dynamics of the lower trophic level of the marine ecosystem. It provides full four-dimensional data for hydrodynamic, organic and inorganic states of the marine ecosystem at a horizontal resolution of roughly $12 \mathrm{~km}$ and at temporal scales of $15 \mathrm{~min}$. In particular it provides fields for average chlorophyll- $a$ concentration and sea-surface temperature, which were used in this study.

To evaluate these model data, two satellite datasets were used:

- Globcolour chlorophyll- $a$ global dataset. This dataset consists of daily chlorophyll- $a$ estimates at a spatial resolution of $\sim 4 \mathrm{~km}$ (based on data from three optical wavelength satellite sensors).

- Pathfinder sea surface temperature (SST) global dataset. This dataset consists of daily sea surface temperature estimates at a spatial resolution of $\sim 4 \mathrm{~km}$ (based on data from a thermal infrared satellite sensor).

For a fair comparison, the region of interest (which is the model domain) is first extracted from the satellite global dataset. The extracted satellite data are then re-gridded to the coarser model grid using a bilinear interpolation.

As suggested by Shutler et al. (2011) we compute the optical depth averaged chlorophyll- $a$ concentration to compare with satellite estimates of chlorophyll- $a$ which are representative of a variable depth depending on the constituent in the water. The model outputs are then cloud-masked on a daily basis using the contemporaneous satellite masks. Finally, monthly composites are created by averaging daily model and satellite data.

We then analyse all data for 2003-2004. The analysis presented hereafter is based on the definition of five quantile ranges as described in Sect. 2.2.1. Each quantile range therefore holds $20 \%$ of the distribution and in Eq. (8) we always have $\varepsilon_{q}=0.2$.

\subsection{Spatio/temporal evaluation of the North East European shelf sea modelling}

Figure 2 shows an example application of the methodology for fields of sea surface temperature (Fig. 2a) and chlorophyll- $a$ concentration (Fig. 2b). Quantiles are reported on the $\mathrm{x}$-axis with the corresponding lower and upper values

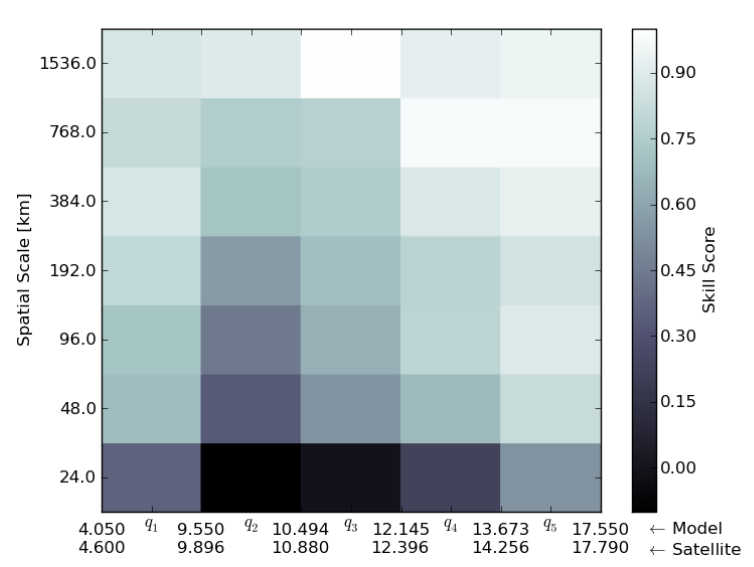

(a)

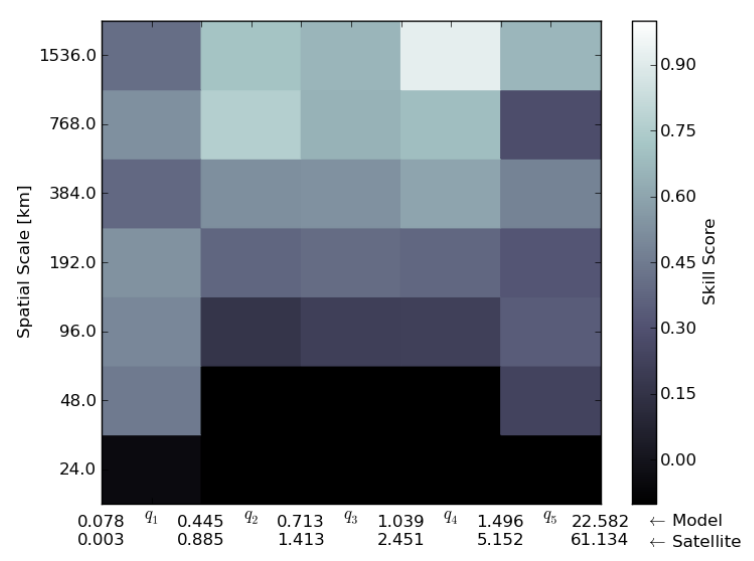

(b)

Fig. 2. Spatial scales versus quantile ranges plots for May 2004 . Sea surface temperature (a) and chlorophyll- $a$ concentration (b) skill scores.

for the satellite and model data. The y-axis shows the spatial scale in kilometres $(\mathrm{km})$.

The methodology highlights scales and ranges of skill. One can notice a lower skill score at small scales $(24 \mathrm{~km})$ for both SST and chlorophyll- $a$ for almost all ranges. One can also note higher model skills for the lowest and the highest quantiles at all spatial scales for SST.

This is less true for chlorophyll- $a$, where a low model skill is observed at high spatial scale (of about $\sim 700 \mathrm{~km}$ ) for the last quantile (high value of chlorophyll- $a$ ). This can be confirmed by looking at the corresponding binary difference map (right-hand map on Fig. 1) where large scale differences are clearly visible in the north of the domain. An interpretation for that observation is a spatial mismatch (or misplacement) of a large summer bloom of chlorophyll- $a$ in the north of the domain.

This methodology also allows us to perform intercomparison of results for different variables and different times (providing they refer to the same geophysical domain). 


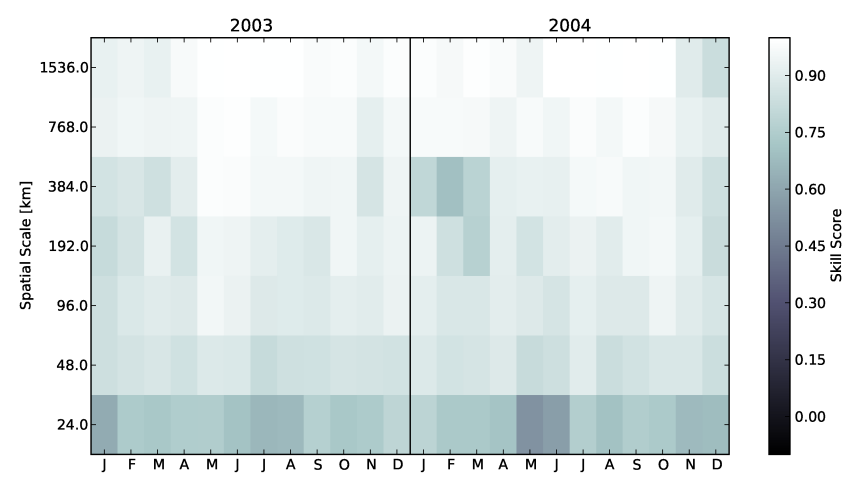

(a)

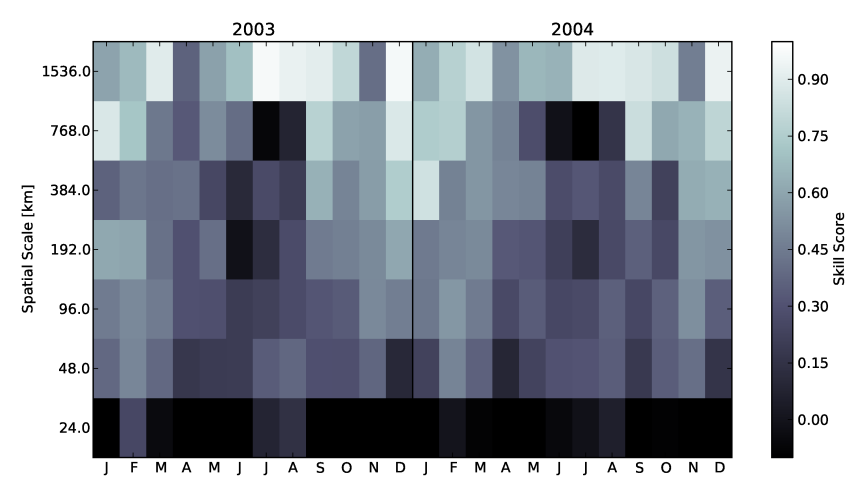

(b)

Fig. 3. Spatial scales versus time plot for the 5th quantile (80$100 \%$ ) 2003-2004. Sea surface temperature (a) and chlorophyll- $a$ concentration (b) skill scores.

Figure 3 shows a time/space skill score plot. Time has been reported on the $x$ axis and spatial scale on the $y$ axis, the shades of grey represent the skill score of the wavelet decomposition for the 5th quantile. The 5th quantile corresponds to the upper range of sea surface temperature and chlorophyll$a$, which in our example can be interpreted as extreme events (i.e. an algal bloom or a temperature anomaly).

Figure $3 \mathrm{a}$ shows that sea surface temperature skill score has high values throughout the year at all spatial scales for the 5th quantile. A small region of slightly lower skill score can be observed during January-March at spatial scales of about $200-400 \mathrm{~km}$. We can also note a slightly lower skill score at low spatial scale throughout the year.

The chlorophyll- $a$ skill score (shown on Fig. 3b) is generally lower than the one of temperature and shows some interesting features. As for the sea surface temperature, we can observe a poorer skill score at low scale (first level of aggregation) throughout the year. However we can additionally observe a consistent patch of low skill in June-August between 100 and $800 \mathrm{~km}$. This pattern does not appear on the temperature skill score.

\subsection{Interpretation of the skill score in terms of model evaluation}

Low skill scores observed at small spatial scales $(\sim 24 \mathrm{~km})$ in both chlorophyll- $a$ and SST model output can be explained by the high small scale variability in the satellite data that is not reproduced by the model. It is indeed easier to capture low frequency variations and trends. Ocean colour and infrared remote sensing are strongly impacted by various sources of uncertainties including measurement noise, calibration noise or atmospheric correction uncertainties. However, these results also illustrate the complexity of modelling biological systems.

The generally higher skill scores obtained for SST, at all scales and for all quantile ranges, (compared with chlorophyll- $a$ ) highlight the strength of the hydrodynamic model fed with high quality surface forcing and boundary conditions (Siddorn et al., 2007). One should also note that chlorophyll- $a$ estimates from ocean colour data are representative of a variable and unknown depth: the water leaving radiances (used to derive chlorophyll- $a$ concentration) include contributions from the surface to a finite depth which varies with the optical properties of the water. For that reason, we choose to average the model chlorophyll- $a$ over the optical depth (of the model), but an uncertainty still remains.

Finally, the consistent appearance of low skill scores in chlorophyll- $a$ (5th quantile) analysis during June-August at large scale is correlated with the summer algal bloom off Scotland and Ireland coast. On the satellite chlorophyll- $a$ field provided on the top-left map of Fig. 1, one can see high chlorophyll- $a$ values $\left(2-8 \mathrm{mg} \mathrm{m}^{-3}\right)$ along the northwest coast of Scotland and Ireland, whereas in the model field, the highest chlorophyll- $a$ values are observed further in the northwest direction and extend further toward the northwest coast of Norway. This translates into the large scale misplacement of pattern visible on the binary difference map (right map on Fig. 1).

\subsection{Discussion}

When comparing model output to another dataset, one may observe differences in the characteristics and possibly in the shape of data distributions. However, the model can still show some skill in representing relative patterns such as extreme events for example. It is therefore important to use a methodology which will be able to highlight the skill of the model without being affected by the bias or the data distribution shape. The bias can be studied separately using simple classical methods but it is worth noting that one can compare the size and mean value of quantile ranges to study it in more detail (for each quantile range separately).

The method presented here allows the comparison of inherent spatial structures within two data sets at different scales. This process is not affected by the overall bias or respective dispersion of the data, as was the case in the original 

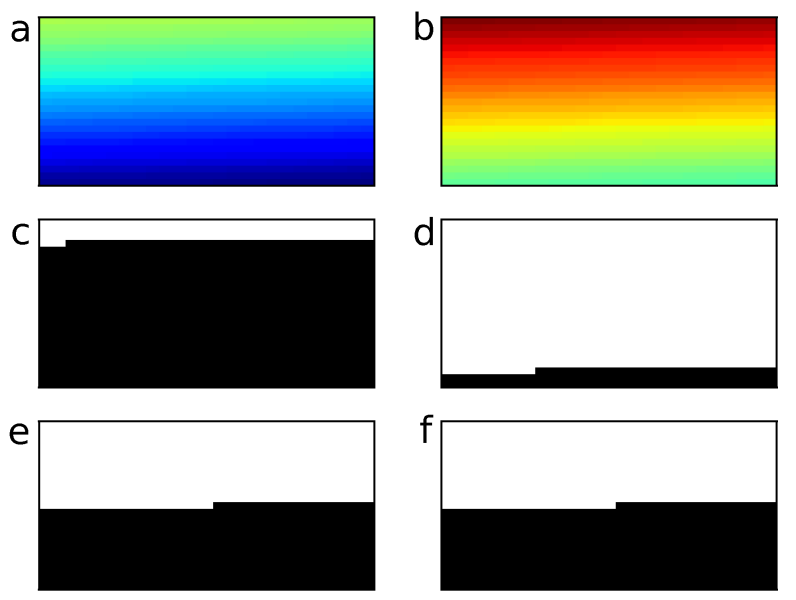

Fig. 4. Illustration of the effect of a bias on the binary masks as defined in Sect. 2.2.1. (a) and (b) are two sample data array where (b) displays a bias with respect to (a). (c) and (d) are the binary masks obtained considering an absolute threshold that is the overall mean value of (a) and (b). (e) and (f) are the binary masks obtained using the quantile approach introduced in Sect. 2.2.1.

version when using absolute thresholds. This is illustrated by Figs. 4 and 5. Starting from two 2-D data arrays that have exactly the same patterns but a systematic difference (bias), Fig. $4 \mathrm{c}$ and dillustrates how using absolute thresholds leads to completely different binary masks. However, using relative thresholds (quantiles) enables the comparison of inherent spatial structures of the data sets (Fig. 4e and f).

Moreover, if we consider two data sets with the same patterns but different distributions (Fig. 5a-d), the binary masks defined by absolute thresholds are very different (Fig. 5e and f) and do not represent comparable structures. The use of quantile definitions provides a more robust definition of the patterns (Fig. 5e and f).

From the two illustrative examples described above, it is clear that if one would use absolute thresholds as break-off criteria for the binary maps, qualitatively similar patterns may appear to be structurally different. An additional benefit of the quantile definition is that it yields the same amount of data points in each quantile range hence guarantees equivalent structural maps.

The wavelet decomposition we described in Sect. 2.2.2 also provides more confidence to the results especially at the higher aggregation levels when comparing data sets with gaps. Applying the original method of Casati et al. (2004) to masked data, a cell (at high aggregation level) that contains very few valid values and a cell containing only valid values would have had the same impact on the overall MSE. The introduction of the weight image $\zeta_{0}$ is a mathematical solution that gives appropriate impact factors to each cell in relation to the data gap contained within it, while preserving the fundamental characteristics of the decomposition, i.e. the orthogonality between the wavelet components and the conservation of the original signal (Eq. 5).
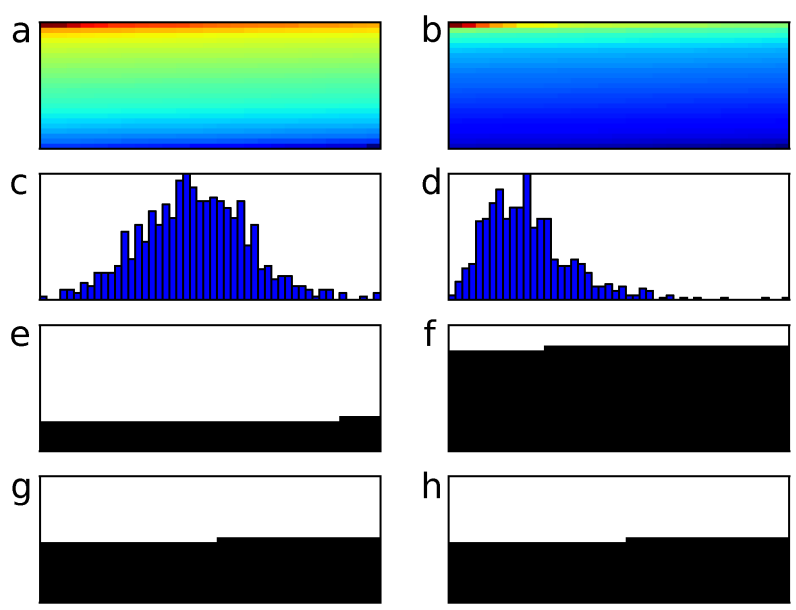

Fig. 5. Illustration of the effect of differences in distributions in the two datasets. (a) and (b) are two sample data arrays where (b) is a power function of (a). (c) and (d) are their respective histograms. (e) and (f) are the binary masks obtained considering an absolute threshold that is the overall mean value of (a) and (b). (g) and (h) are the binary masks obtained using the quantile approach introduced in Sect. 2.2.1, i.e. the median in this case.

This methodology is based on statistically robust metrics and the choice of the threshold is driven by the data distribution, and hence is more objective (for example this allows the study of patterns of extreme events of chlorophyll- $a$ ) in comparing the inherent structures of the datasets. This is particularly useful for temporal intercomparisons ie for situations where the bias in different time series is potentially different.

\section{Conclusions}

The approach presented here has been developed to compare the spatial structures in two datasets. It allows any spatial differences to be decomposed into their orthogonal components. The method is composed of two steps: (i) definition of binary error map based on quantile classification (ii) wavelet decomposition of the binary error map and computation of a skill score for each level of decomposition. The approach is generic in the sense that it requires no tuning or parameter selection as thresholding to generate the binary difference maps is determined based on the statistical distribution of the input datasets. Furthermore, the approach is able to handle data containing biases or occluded (missing) data, without loss of orthogonality. We have demonstrated its application by analysing a series of scenes of model output with optical wavelength satellite data. The methodology provides the ability to identify the spatial scales of the features that the model is able to reproduce focusing on the inherent structures of the datasets independently of bias or normalised standard deviation. 
The results can be visualised in two very synthetic ways: a spatial scales versus quantile ranges plot, which can be used to identify the overall match/mismatch of the features in two 2-D data fields; and a spatial scales versus time plot, which can be used to analyse extreme events. Alternatively, if one is interested in a specific spatial scale, a time/quantile plot would provide useful information over the whole data range.

This methodology, used in combination with other classical ways of comparing two datasets, is a powerful evaluation tool (when comparing Earth observation data and model output) because it is objective and independent of the dataset distribution. It is therefore a very useful tool that can serve to justify or guide the choice of a model for a specific application. In the context of marine hydrological/ecosystem modelling these can be carbon budget, harmful algal bloom detection, ecosystem management.

One can also use this methodology as a way of comparing outputs from two different model. The method provides a synthetic way of representing the spatial effect of two different parametrisations, or the effect of using different boundary conditions or forcing data in terms of spatial features.

Future work will concentrate on extending the approach to include the time dimension. This would enable a complete picture of the model skill to be considered including seasonal forecasts and the study of inter-annual or multi-decadal trends.

\section{Supplementary material related to this article is available online at: http://www.geosci-model-dev.net/5/223/2012/ gmd-5-223-2012-supplement.zip.}

Acknowledgements. The authors would like to thank MyOcean project for supplying Globcolour data for this study. The AVHRR Oceans Pathfinder SST data were obtained from the Physical Oceanography Distributed Active Archive Center (PO.DAAC) at the NASA Jet Propulsion Laboratory, Pasadena, CA. http://podaac.jpl.nasa.gov. The authors thank the NERC Earth Observation Data Acquisition and Analysis Service (NEODAAS) for providing computing facilities and data storage. This work was funded by the UK NERC Oceans 2025 program theme 9, Next Generation Ecosystem Models. This work was partially supported by the EC FP7 MyOcean research and development project "Improving $\mathrm{CO}_{2}$ Flux Estimations from the MyOcean Atlantic north west shelf hydrodynamic ecosystem model (IFEMA)".

Edited by: J. Annan

\section{References}

Allen, J. I., Blackford, J. C., Holt, J. T., Proctor, R., Ashworth, M., and Siddorn, J. R.: A highly spatially resolved ecosystem model for the North West European Continental Shelf, Sarsia, 86, 423440, 2001.
Allen, J. I., Somerfield, P., and Gilbert, F.: Quantifying uncertainty in high-resolution coupled hydrodynamic-ecosystem models, J. Marine Syst., 64, 3-14, doi:10.1016/j.jmarsys.2006.02.010, 2007.

Bougeault, P.: The WGNE survey of verification methods for numerical prediction of weather elements and severe weather events, Tech. rep., Technical report, WMO, 2003.

Briggs, W. M. and Levine, R. A.: Wavelets and field forecast verification, B. Am. Meteor. Soc., 125, 1329-1341, 1997.

Casati, B.: New Developments of the Intensity-Scale Technique within the Spatial Verification Methods Intercomparison Project, Weather Forecast., 25, 113-143, doi:10.1175/2009WAF2222257.1, 2010.

Casati, B., Ross, G., and Stephenson, D.: A new intensity-scale approach for the verification of spatial precipitation forecasts, Meteorol. Appl., 11, 141-154, 2004.

Doney, S. C., Lima, I., Moore, J. K., Lindsay, K., Behrenfeld, M. J., Westberry, T. K., Mahowald, N., Glover, D. M., and Takahashi, T.: Skill metrics for confronting global upper ocean ecosystembiogeochemistry models against field and remote sensing data, J. Marine Syst., 76, 95-112, 2009.

Ebert, E. E., Damrath, U., Wergen, W., and Baldwin, E.: The WGNE assessment of short-term quantitative precipitation forecasts (QPFs) from operational numerical weather prediction models, B. Am. Meteor. Soc., 84, 481-492, 2003.

Haar, A.: Zur Theorie der orthogonalen Funktionensysteme, Math. Ann., 69, 331-371, 1910 (in German).

Jones, A., Thomson, D., Hort, M., and Devenish, B.: The UK Met Office's Next-Generation Atmospheric Dispersion Model, NAME III, NATO Challenges of Modern Series, 580-589, 2007.

Lehr, W., Wesley, D., Simecek-Beatty, D., Jones, R., Kachook, G., and Lankford, J.: Algorithm and interface modifications of the NOAA oil spill behavior model, in: Proceedings of the TwentyThird Arctic and Marine Oilspill Program (AMOP) Technical Seminar, 525-540, 2000.

Shutler, J., Smyth, T., Saux Picart, S., Wakelin, S., Hyder, P., Grant, M., Orekhov, P., Tilstone, G., and Allen, J. I.: Evaluating the ability of a hydrodynamic ecosystem model to capture inter- and intra-annual spatial characteristics of chlorophyll$\mathrm{a}$ in the north east Atlantic, J. Marine Syst., 88, 169-182, doi:10.1016/j.jmarsys.2011.03.013, 2011.

Siddorn, J. R., Allen, J. I., Blackford, J. C., Gilbert, F. J., Holt, J. T., Hort, M. W., Osborne, J. P., Proctor, R., and Mills, D. K.: Modelling the hydrodynamics and ecosystem of the North-West European continental shelf for operational oceanography, J. Marine Syst., 65, 417-429, doi:10.1016/j.jmarsys.2006.01.018, 2007.

Stow, C. A., Jolliff, J., McGillicuddy Jr., D. J., Doney, S. C., Allen, J. I., Friedrichs, M. A., Rose, K. A., and Wallhead, P.: Skill assessment for coupled biological/physical models of marine systems, J. Marine Syst., 76, 4-15, 2009.

Tiedje, B., Moll, A., and Kaleschke, L.: Comparison of temporal and spatial structures of chlorophyll derived from MODIS satellite data and ECOHAM3 model data in the North Sea, J. Sea Res., 64, 250-259, 2010.

Yates, E., Anquetin, S., Ducrocq, V., Creutin, J.-D., Ricard, D., and Chancibault, K.: Point and areal validation of forecast precipitation fields, Meteorol. Appl., 13, 1-20, doi:10.1017/S1350482705001921, 2006. 